\title{
Sulfonic acid functionalized mesoporous MCM-41 silica as a convenient catalyst for Bisphenol-A synthesis
}

\author{
Debasish Das, ${ }^{a}$ Jyh-Fu Lee ${ }^{b}$ and Soofin Cheng*a \\ a Department of Chemistry, National Taiwan University, Taipei 106, Taiwan. \\ E-mail:chem1031@ccms.ntu.edu.tw \\ ${ }^{b}$ Research Division, Synchrotron Radiation Research Center, Hsinchu 300, Taiwan
}

\author{
Received (in Cambridge, UK) 7th August 2001, Accepted 18th September 2001 \\ First published as an Advance Article on the web 5th October 2001
}

\begin{abstract}
Sulfonic acid groups anchored to the surface of mesoporous MCM-41 silica have been identified with S K-edge XANES spectra and the material is an efficient catalyst for the liquid phase condensation of phenol with acetone to form Bisphenol-A with high selectivity.
\end{abstract}

We report here that sulfonic acid functionalized mesoporous MCM-41 silica can be an efficient catalyst for the condensation of phenol and acetone at relatively low temperature to synthesize Bisphenol-A with a very high selectivity. Bisphenol$\mathrm{A}$ is an important raw material for polymer and resin production, and it is produced industrially using ion-exchange resins such as Amberlyst. ${ }^{1}$ However, thermal stability and fouling of the resins are major problems for these catalysts and the search for thermally stable and regenerable solid acid catalysts are continuing.

Zeolites have been increasingly used in the synthesis of fine chemicals due to their high surface area and confined domains. ${ }^{2}$ However, the suitability of zeolites for the conversion of larger substrates has been severely limited by the available dimensions of their pores, which are in the micropore region with pore mouth diameter usually less than $1 \mathrm{~nm} .{ }^{3}$ For reactions to occur within the pores, the reaction substrates and products must be allowed to diffuse smoothly through the zeolite pores. The discovery of surfactant-templated mesoporous silica materials such as MCM-41 (hexagonal P6m symmetry) and MCM-48 (cubic Ia3d symmetry) with controlled pore diameters (2-10 $\mathrm{nm})$ and topologies has extended the suitability of these materials for the conversion of larger substrates. ${ }^{4}$ They are very promising for the application as catalysts and/or catalyst supports and are likely to offer potential improvements in reaction selectivity in the conversion of larger substrate molecules in their well-defined channels with narrow pore size distributions. However, in spite of these favorable pore dimensions, the acidity of Al-substituted mesoporous materials such as Al-MCM-41, is much weaker than that of microporous zeolites. ${ }^{5}$ To overcome this drawback, the synthesis of inorganic-organic hybrid mesoporous materials with alkylsulfonic acid groups has been reported recently. ${ }^{6-9}$ These hybrid materials can be synthesized either by direct one-step synthesis or via secondary silylation. Thus, $\mathrm{MCM}-\mathrm{SO}_{3} \mathrm{H}$ prepared by direct synthesis and secondary silyation is reported to be highly efficient in the synthesis of bisfurylalkanes ${ }^{6}$ and also in the esterification of glycerol with fatty acids. ${ }^{7,9}$ However, as the organic functional groups may be damaged or destroyed during the template removal process, the secondary silylation technique seems to offer a better alternative for preparing mesoporous acid catalysts.

Pure silica MCM-41 was synthesized according to the method described earlier. ${ }^{10}$ Surface functionalization with sulfonic acid groups was carried out according to the method described in the literature. ${ }^{7,8}$ Typically, $3.0 \mathrm{~g}$ of the freshly calcined MCM-41 sample was evacuated at $150{ }^{\circ} \mathrm{C}$ and then an excess of 3-mercaptopropyltrimethoxysilane (MPTS) in dry toluene was introduced. The mixture was refluxed for $6 \mathrm{~h}$ and the solid was filtered off, washed with dry toluene and air-dried.
The $-\mathrm{SH}$ groups were converted into $-\mathrm{SO}_{3} \mathrm{H}$ groups by mild oxidation with $\mathrm{H}_{2} \mathrm{O}_{2}$ (stirring for $24 \mathrm{~h}$ at $60{ }^{\circ} \mathrm{C}$ with excess oxidant). The solid was filtered off, washed with water and ethanol, followed by acidification with $0.1 \mathrm{M} \mathrm{H}_{2} \mathrm{SO}_{4}$ followed by thorough washing with water to remove all traces of liquid acid. The solid material was finally dried at $60{ }^{\circ} \mathrm{C}$ overnight.

Sulfur loading of the solid material was determined by elemental analysis (Heraeus) and was typically in the range $0.9-1.8$ meq. $\mathrm{g}^{-1}$ of solid. Thermogravimetric analysis (Du Pont 950) shows the material to be more hydrophobic than MCM-41. While calcined pure silica MCM-41 shows ca. $5 \%$ weight loss in the range 30-100 due to water removal, sulfonic acid functionalized MCM-41 (denoted $\mathrm{MCM}-\mathrm{SO}_{3} \mathrm{H}$ ) showed only $<1 \%$ weight loss in this region. Loss of sulfonic acid groups starts at $\mathrm{ca} .200{ }^{\circ} \mathrm{C}$ and is completed by $325^{\circ} \mathrm{C}$. The specific surface area and pore volume of the pure silica MCM41 material was reduced from $1035 \mathrm{~m}^{2} \mathrm{~g}^{-1}$ and $0.85 \mathrm{~cm}^{3} \mathrm{~g}^{-1}$, respectively, to $690 \mathrm{~m}^{2} \mathrm{~g}^{-1}$ and $0.42 \mathrm{~cm}^{3} \mathrm{~g}^{-1}$ after anchoring the sulfonic acid groups. Although the pore size distribution curve (Fig. 1) becomes somewhat broad after sulfonic acid group introduction and the peak maximum shifts from $27 \AA$ (pure MCM-41) to $22 \AA$, the pore size distribution still remains very narrow with major fractions of pores lying within the $7 \AA$ region. These results indicate that anchoring sulfonic groups to the channels of MCM-41 reduced the pore diameter and volume of MCM-41 but did not damage the mesoporous structure. This was also affirmed by the retention of sharp diffraction peaks in the corresponding XRD pattern.

To obtain good catalytic activity it is imperative that the thiol groups can be effectively oxidized to active sulfonic acid groups. To monitor this process, the oxidation states of sulfur were examined by studying the S K-edge XANES spectra of the sample before and after oxidation. The XANES experiments were performed at Beam line $15 \mathrm{~B}$ at the Synchrotron Radiation Research Center facility at Hsinchu, Taiwan. Standard operating conditions were $1.5 \mathrm{GeV}$ and $200 \mathrm{~mA}$ beam current and the

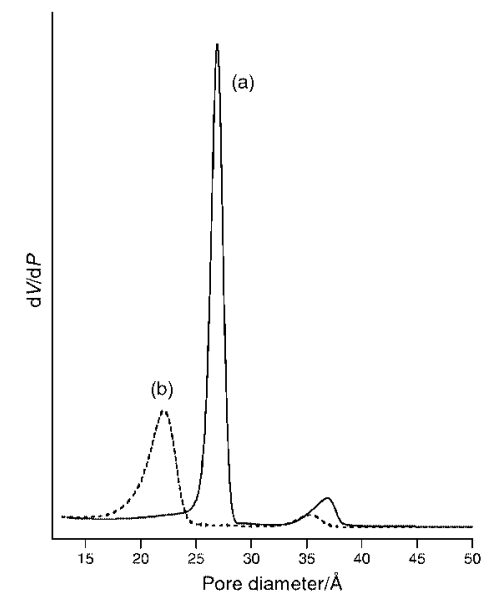

Fig. 1 Pore size distribution of (a) calcined pure silica MCM-41 and (b) MCM-41 functionalized with sulfonic acid groups. 
photon energies were calibrated using the L-edge of pure Mo foil. Fig. 2 shows the S K-edge XANES spectra of the thiol functionalized samples before and after $\mathrm{H}_{2} \mathrm{O}_{2}$ oxidation. It has been reported that both the energy position $\left(E_{\mathrm{k}}\right)$ and intensity of the $S \mathrm{~s} \rightarrow \mathrm{p}$ transition peak (white-line) are sensitively related to the oxidation state of the $\mathrm{S}$ atom. ${ }^{11}$ Studies with model compounds showed an increase of $E_{\mathrm{k}}$ and amplitude of the white-line with increasing formal oxidation states of S. Thus, for unoxidized sample containing thiol groups the white-line appears at $2472 \mathrm{eV}$ corresponding to sulfur in the reduced state. After $\mathrm{H}_{2} \mathrm{O}_{2}$ oxidation the white-line shifts to higher energy $(2481 \mathrm{eV})$ which corresponds to sulfonic acid with $\mathrm{S}$ in the +5 state. ${ }^{11,12}$ It can be seen from Fig. 2 that most of the anchored thiol groups have been oxidized to sulfonic acid groups under the above oxidation conditions.

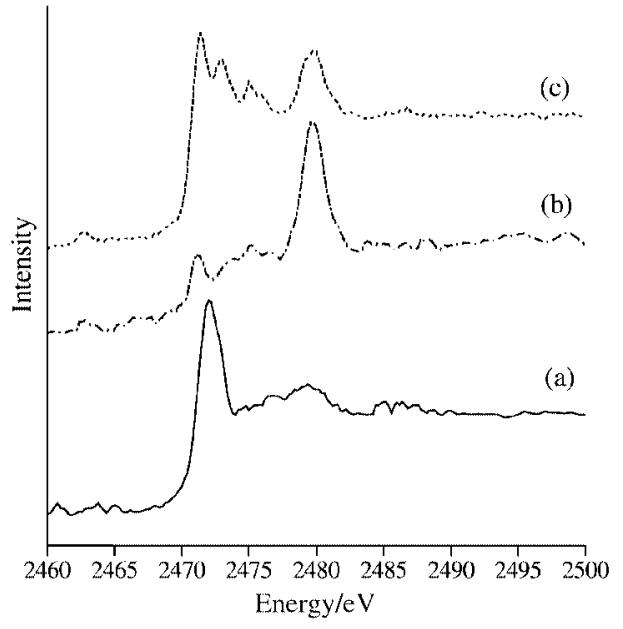

Fig. 2 S K-edge XANES spectra of thiol functionalized MCM-41 materials (a) before oxidation, (b) after oxidation and (c) sample with incomplete oxidation of thiol groups.

Condensation of phenol and acetone to Bisphenol-A (Scheme 1) was carried out at temperatures of $70-125{ }^{\circ} \mathrm{C}$ with the sulfonic acid functionalized MCM-41 silicas. Several other acidic zeolites such as H-ZSM-5, H-Y and H-beta have also been used for comparison. The products were analyzed and identified using a Chrompak 9000 gas chromatograph and HP 6890 GC-MS, and the results are given in Table 1. No conversion of phenol was observed in the absence of catalyst. While acidic zeolites like H-ZSM-5, H-Y and H-beta showed negligible activity at $70{ }^{\circ} \mathrm{C}, \mathrm{MCM}-\mathrm{SO}_{3} \mathrm{H}$ showed $30 \%$ phenol conversion ( $c f$. $40 \%$ maximum theoretical conversion at a phenol:acetone molar ratio of $5: 1)$ with $>90 \%$ selectivity towards $p, p^{\prime}$-Bisphenol-A; the other product was $o, p^{\prime}$-Bisphenol-A. The conversion was found to increase with the reaction temperature, but the selectivity remained almost unchanged up to $120{ }^{\circ} \mathrm{C}$. Chroman and trisphenols were not detected at temperature below $125{ }^{\circ} \mathrm{C}$, but small amounts $(<1 \%)$ of these byproducts were detected at or above $125^{\circ} \mathrm{C}$. Recently, the synthesis of Bisphenol-A over heteropolyacid encapsulated MCM-41 has been reported. ${ }^{13}$ However, 12 -tungstophosphoric acid encapsulated MCM-41 was found to be catalytically active only at temperatures above $120^{\circ} \mathrm{C}$. Due to this high reaction temperature, several by-products such as alkylated phenols and chroman derivatives were formed, and

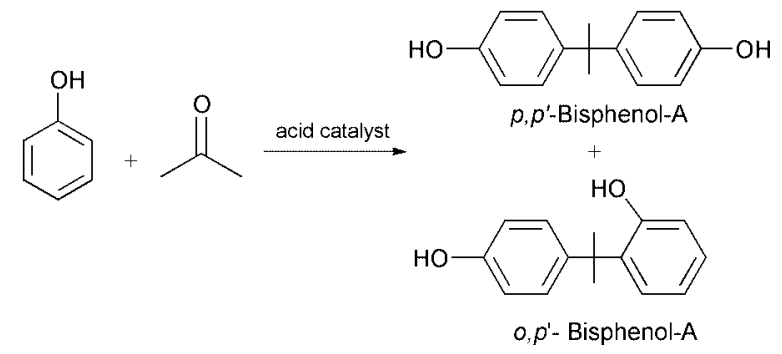

Scheme 1
Table 1 Synthesis of Bisphenol-A with sulfonic acid functionalized MCM41 silicas $^{a}$

\begin{tabular}{llll}
\hline Entry & Catalyst & $\begin{array}{l}\text { Phenol } \\
\text { conversion }(\%)\end{array}$ & $\begin{array}{l}\text { Selectivity }^{b} \\
(\%)\end{array}$ \\
\hline 1 & - & 0 & - \\
2 & H-beta $^{c}$ & 5 & 55 \\
3 & $\mathrm{HY}^{d}$ & 7 & - \\
4 & $\mathrm{HZSM}^{2}$ & $<5$ & 10 \\
5 & ${\mathrm{MCM}-\mathrm{SO}_{3} \mathrm{H}}_{6}$ & 30 & 92 \\
$\mathrm{MCM}^{-} \mathrm{SO}_{3} \mathrm{H}^{f}$ & 17 & 90
\end{tabular}

a Reagents and conditions: Phenol $4.7 \mathrm{~g}$, acetone $0.58 \mathrm{~g}$, (molar ratio = 5:1), catalyst $50 \mathrm{mg}, 70{ }^{\circ} \mathrm{C}, 24 \mathrm{~h} .{ }^{b}$ Selectivity to $p, p^{\prime}$-Bisphenol-A. ${ }^{c} \mathrm{Si} / \mathrm{Al}$ $=50 .{ }^{d} \mathrm{Si} / \mathrm{Al}=10.6 .{ }^{e} \mathrm{Si} / \mathrm{Al}=80 .{ }^{f}$ Incomplete oxidation (from XANES).

the selectivity to Bisphenol-A was much lower than that obtained with $\mathrm{MCM}-\mathrm{SO}_{3} \mathrm{H}$. Furthermore, leaching of sulfur during the catalytic reaction was found to be negligible with $\mathrm{MCM}-\mathrm{SO}_{3} \mathrm{H}$. Elemental analysis of the catalyst before reaction indicated $\mathrm{ca}$. $3.4 \%$ sulfur and $5.6 \%$ carbon content. After reaction at $70{ }^{\circ} \mathrm{C}$, these values were $3.2 \% \mathrm{~S}$ and $9.5 \% \mathrm{C}$. The slight decrease in sulfur content is mainly due to the increase in weight from adsorption of organic species. On the other hand, it is noted that optimization of the oxidation process in the preparation of $\mathrm{MCM}-\mathrm{SO}_{3} \mathrm{H}$ is essential to achieve high catalytic activity. For samples with similar sulfur loadings but with incompletely oxidized thiol groups (dotted line in Fig 2) the activity was found to be much lower (see Table 1). XANES spectra of the samples with incompletely oxidized sulfur species showed a number of peaks around $2472 \mathrm{eV}$, suggesting the presence of lower valent sulfur species such as sulfides and disulfides. ${ }^{11,12}$ It has also been observed that at higher sulfur loadings ( $>1.5$ meq. $\mathrm{g}^{-1}$ solid) part of the sulfur remains in the reduced form even after prolonged oxidation, probably due to formation of sulfides and disulfides.

In conclusion, sulfonic acid anchored MCM-41 has been found to be very effective in the synthesis of $p, p^{\prime}$-Bisphenol-A with very high selectivity at relatively low reaction temperatures. S K-edge XANES studies can be used as a convenient tool for easy monitoring of the oxidation state of sulfur in the samples.

Financial support was provided by the Chinese Petroleum Corporation and the Ministry of Education, Taiwan.

\section{Notes and references}

1 G. D. Yadov and N. Kirthivasan, Appl. Catal., 1997, 154, 29.

2 M. E. Davis, Microporous Mesoporous Mater., 1998, 21, 173; R. A. Sheldon, J. A. Elings, S. K. Lee, H. E. B. Lempers and R. S. Downing, J. Mol. Catal. A, 1998, 134, 129; S. Feast and J. A. Lercher, Stud. Surf. Sci. Catal., 1996, 102, 363.

3 M. E. Davis, Acc. Chem. Res., 1993, 26, 111.

4 J. S. Beck, J. C. Vartuli, W. J. Roth, M. E. Leonowicz, C. T. Kresge, K. D. Schmitt, C. T. Chu, D. H. Olson, E. W. Sheppard, S. B. McCullen J. B. Higgins and J. L. Schlenker, J. Am. Chem. Soc., 1992, 114, 10834; P. T. Tanev and T. J. Pinnavaia, Science, 1995, 267, 865; X. S. Zhao, G. Q. Lu and G. J. Miller, Ind. Eng. Chem. Res., 1996, 35, 2075.

5 A. Corma, V. Fornes, M. T. Navarro and J. Perez-Pariente, J. Catal., 1994, 148, 569.

6 W. Van Rhijn, D. De Vos, W. Bossert, J. Bullen, B. Wouters, P. Grobet and P. Jacobs, Stud. Surf. Sci. Catal., 1998, 117, 183; W. M. Van Rhijn, D. E. B. F. Sels, W. D. Bossert and P. A. Jacobs, Chem. Commun., 1998 317.

7 W. D. Bossert, D. E. De vos, W. M. Van Rhijn, J. Bullen, P. J. Grobet and P. A. Jacobs, J. Catal., 1999, 182, 156.

8 M. H. Lim, C. F. Blanford and A. Stein, Chem. Mater., 1998, 10, 467.

9 I. Diaz, F. Mohino, J. Perez-Pariente and E. Sastre, Appl. Catal. A, 2001, 205, 19.

10 D. Das, C-M. Tsai and S. Cheng, Chem. Commun., 1999, 473.

11 G. Sarret, J. Connan, M. Kasrai, G. M. Bancroft, A. Charrie-Duhaut, S. Lemoine, P. Adam, P. Albrecht and L. Eybert-Berard, Geochim. Cosmochim. Acta., 1999, 63, 3767.

12 K. Xia, F. Weesner, W. F. Bleam, P. R. Bloom, U. L. Skyllberg and P. A. Helmke, Soil Sci. Soc. Am. J., 1998, 62, 1240.

13 K. Nowinska and W. Kaleta, Appl. Catal. A, 2001, 203, 91. 